\title{
A HOUSEHOLD SIR EPIDEMIC MODEL INCORPORATING TIME OF DAY EFFECTS \\ PETER NEAL, ${ }^{*}$ Lancaster University
}

\begin{abstract}
During the course of a day an individual typically mixes with different groups of individuals. Epidemic models incorporating population structure with individuals being able to infect different groups of individuals have received extensive attention in the literature. However, almost exclusively the models assume that individuals are able to simultaneously infect members of all groups, whereas in reality individuals will typically only be able to infect members of any group they currently reside in. In the current work we develop a model where individuals move between a community and their household during the course of the day, only infecting within their current group. By defining a novel branching process approximation with an explicit expression for the probability generating function of the offspring distribution, we are able to derive the probability of a major epidemic outbreak.

Keywords: Birth-death process; branching processes; households SIR epidemic model
\end{abstract}

2010 Mathematics Subject Classification: Primary 92D30

Secondary 60J80;60G10

\section{Introduction}

The type of contacts made by an individual depend upon the time of day. For example, during the day an individual might be in contact with work colleagues or fellow school pupils and in the evening return home to their family. Therefore the group of individuals whom an infectious individual can infect varies during the day. However, mathematical models for infectious disease spread have not taken into account the changing group of contacts during the day. Over the past twenty years there has

\footnotetext{
* Postal address: Department of Mathematics and Statistics, Fylde College, Lancaster University,
} LA1 4YF, United Kingdom 
been considerable interest in incorporating population structure into epidemic models, see, for example, Ball et al. (1997), Ball and Neal (2002), Ball and Neal (2008). In all of the above mentioned papers individuals are assumed to make infectious contacts with different groups of individuals, typically at different rates. However, it is assumed that infectives are able to simultaneously infect members from different groups, for example, work colleagues and family members. This is clearly an unrealistic assumption.

The aim of the current work is to explicitly model individuals moving between different contact groups during the course of a day. In particular, we develop a household model where individuals spend the daytime mixing as a community before returning to their families (households) for the nighttime. The type of infectious contacts an individual makes depends on the time of day. A novel branching process approximation for the initial stages of the epidemic process is derived. The branching process approximation is a multitype Galton-Watson branching process based on the infectious status of households at the start of each day as classical branching process approximations for household epidemics based on Ball (1996) and Ball et al. (1997) are problematic in the current setup.

The remainder of the paper is structured as follows. In Section 2, the model is described along with the approximating branching process. In Section 3, an explicit expression for the probability generating function of the offspring distribution of the branching process is derived. In Section 4, we study special cases of the epidemic model, where explicit expressions for the probability of a major epidemic can be obtained. These include the non-household model (all households are of size 1) and highly infectious households (an infective instantaneously infects all members of their household on returning to their household), where in both cases the approximating branching process can be reduced to a single type Galton-Watson branching process. Finally, in Section 5 we present numerical results and show how the approach taken in Section 3 can be used to obtain extinction probabilities in situations where it is difficult to derive an analytical explicit expression for the probability generating function of the offspring distribution. 


\section{Model and branching process approximation}

We consider the spread of an SIR epidemic, $\mathcal{E}_{N}$ say, among a population of $N$ individuals living in $n$ households. The population is partitioned into households. For $k=1,2, \ldots$, let $q_{k}^{N}$ denote the proportion of individuals who belong to a household of size $k$. Time is divided into days and the basic unit of time is one day. Each day consists of two periods which we shall term morning and night. The length of the morning period, which takes place at the start of each day, is $\tau(0<\tau \leq 1)$ with correspondingly the night period being of length $1-\tau$. During the morning the whole population is mixing together, whilst at night the individuals return to their households. Whilst infectious, an infective can make infectious contact with any member of the population during the morning, but at night they can only infect members of their own household.

The epidemic is defined as follows. For $i=1,2, \ldots, n$, let $h_{i}$ denote the total size of household $i$ and for $j=1,2, \ldots, h_{i}$, let $(i, j)$ denote the $j^{\text {th }}$ individual in household $i$. Let $Q_{i, j}$ denote the length of individual $(i, j)$ 's infectious period if he or she becomes infected. We assume that the infectious periods are independent and identically distributed and thus do not depend upon the time of day at which an individual is infectious. During the morning infectious individuals make infectious contacts at the points of homogeneous Poisson point processes having rate $\lambda$ with the individual contacted chosen uniformly at random from the whole population. During the night infectious individuals make infectious contacts at the points of independent Poisson processes having rate $\beta$ with each other member of their household. Thus if an individual belongs to a household of size $h$ the total rate at which he or she is making infectious contacts during the night is $(h-1) \beta$. If a contacted individual is susceptible then he/she becomes infected and is immediately able to infect other susceptibles. Thus there is no latent period, although we briefly discuss the inclusion of a latent period briefly in Section 5. An infected individual is removed at the end of his/her infectious period and plays no further part in the epidemic. The epidemic is assumed to start with a single infective at the start of a day (morning), although this can easily be relaxed to allow the initial infective to become infectious at anytime of day. 
The focus of this paper is the initial stages of the epidemic and answering the question of; what is the probability of a major epidemic outbreak? In order to make this question precise, we consider the limiting behaviour of $\mathcal{E}_{N}$ as $N \rightarrow \infty$. In particular, we construct a branching process approximation for the epidemic with the probability that the branching process does not go extinct corresponding to the probability of a major epidemic outbreak. Branching process approximations for epidemic models have a long and illustrious history dating back to Whittle (1955). The approach taken in Whittle (1955) and subsequently in Ball (1983) and Ball and Donnelly (1995) for the homogeneously mixing epidemic model is to couple the epidemic to a branching process so that infectives in the epidemic correspond to individuals in the branching process. Thus equating infectious period and infectious contacts in the epidemic to lifetime and births in the branching process, respectively, the two processes can be coupled so that there are the same number of individuals in both processes until the first time at which an attempt is made to infect a previously infected individual in the epidemic process. This does not occur until $O(\sqrt{N})$ of the population have been infected, see Ball and Donnelly (1995), and is linked to the classic birthday problem, see, for example, Aldous (1985). Thus for large $N$, the epidemic has either died out (branching process goes extinct) or has become established (a major outbreak has occurred) before the coupling breaks down. Branching process approximations for household epidemics are established in Ball (1996) and Ball et al. (1997). Since there is a high probability of repeated infectious contacts between individuals belonging to the same household, infectious households (those households with at least one infectious individual) are coupled to individuals in a branching process with between household (global infections) coupled to births in the branching process. A similar approach has been taken to establish branching process approximations for the great circle epidemic model, Ball and Neal (2003), and for network epidemic models, Ball and Neal (2008).

A branching process approximation with individuals corresponding to infectious households in the manner of Ball (1996) and Ball et al. (1997) is not helpful for the current model. The reason for this is that the infectious behaviour of a household depends upon the time of day at which the first member of the household becomes infected. Thus we would require an approximating continuous state branching process, see, for example, Lamperti (1967), with type indexed by the time of day at which 
the household first became infected. Our alternative solution is for individuals in the branching process to correspond to infectious households in the epidemic at the start of each day. That is, we couple the status of the epidemic at the start of each day to a multitype Galton-Watson branching process, where the infectious households are classified into type by the number of infectives and susceptibles that belong to the household. Therefore a type $(i, j)$ household has $i$ infectives and $j$ susceptibles and we couple this household to a type $(i, j)$ individual in the approximating branching process. The coupling is given in detail in Section 3.

The key assumption of the branching process approximation is that during the morning all infectious contacts (births in the branching process) are with individuals who belong to susceptible households (no member of the household has previously been infected). It is straightforward to show that if the maximum household size is finite that no global infectious contact takes place with a previously infected household until $O(\sqrt{N})$ of the population have been infected, as in the homogeneously mixing case. This can be made fully rigorous with a coupling argument along the lines of Ball and Donnelly (1995) and Ball and Neal (2003). We will omit the details and instead focus on the approximating branching process.

\section{Probability of a major epidemic outbreak}

In this section we derive an explicit expression for the probability generating function of the offspring distribution of the approximating branching process. In order to make analytical progress we restrict attention to $Q \sim \operatorname{Exp}(\gamma)$ and exploit the Markov structure of the resulting model. Numerical extensions of the results obtained in this section are given in Section 5 using the method of stages Barbour (1976).

For $i, k \in \mathbb{N}$ and $j, l \in \mathbb{Z}^{+}$, let $\mathbf{X}_{(i, j)}$ denote the offspring distribution vector of a household starting a day with $i$ infectives and $j$ susceptibles, where $X_{(i, j),(k, l)}$ is the total number of households of type $(k, l)$ at the end of the day originating from a single household of type $(i, j)$. It is convenient to break the day into morning and night when considering the offspring distribution. During the morning the $i$ infectives in the household behave independently making infectious contacts throughout the entire population and since $Q \sim \operatorname{Exp}(\gamma)$, the infectious process can be coupled to a birth- 
death process with birth rate $\lambda$ and death rate $\gamma$. The main consideration with the coupling to a birth-death process is that it is necessary for us to distinguish between the initial infectives and those infected during the morning who (with probability tending to 1 as $N \rightarrow \infty$ ) will all belong to distinct households. Given that the $i$ infectives behave in an independent and identically distributed manner during the morning we start by considering the morning offspring from a single infective.

Consider a birth-death process with birth and death rates $\lambda$ and $\gamma$, respectively, started with one individual at time 0 . Let $Z(t)$ denote the total number of individuals alive in the birth-death process at time $t$. Let $I(t)=1$ if the initial individual is alive at time $t$ and $I(t)=0$ otherwise. Let $Y(t)=Z(t)-I(t)$, the total number of individuals alive in the branching process at time $t$, excluding the initial individual. The key quantities of interest are $\varphi_{k}(s ; t)=\mathbb{E}\left[s^{Y(t)} 1_{\{I(t)=k\}}\right]$ for $k=0,1,0 \leq s \leq 1$ and $t \geq 0$.

Lemma 3.1. For $0 \leq s \leq 1$ and $t \geq 0$,

$$
\varphi_{0}(s ; t)= \begin{cases}\frac{\gamma(1-s)-(\gamma-\lambda s) \exp (\{\gamma-\lambda\} t)+s(\gamma-\lambda) \exp (-\lambda t)}{\lambda(1-s)-(\gamma-\lambda s) \exp (\{\gamma-\lambda\} t)} & (\lambda \neq \gamma) \\ \frac{\lambda t+s\{1-\lambda t-\exp (-\lambda t)\}}{1+\lambda t(1-s)} & (\lambda=\gamma)\end{cases}
$$

and

$$
\varphi_{1}(s ; t)= \begin{cases}\frac{(\lambda-\gamma) \exp (-\lambda t)}{\lambda(1-s)-(\gamma-\lambda s) \exp (\{\gamma-\lambda\} t)} & (\lambda \neq \gamma) \\ \frac{\exp (-\lambda t)}{1+\lambda t(1-s)} & (\lambda=\gamma)\end{cases}
$$

Proof. Let $\mathcal{B}$ denote an immigration-birth-death process, where the immigration and birth rates are both $\lambda$ and the death rate is $\gamma$. Let $B(t)$ denote the total number of individuals alive in $\mathcal{B}$ at time $t$ and suppose that $B(0)=0$. The immigration behaviour is equivalent to having an individual alive throughout the process giving birth to immigrants. Therefore in the case where $I(t)=1$, the initial individual is alive throughout the period $[0, t]$ and $\{Y(t) \mid I(t)=1\} \stackrel{D}{=} B(t)$. From Gani and Stals (2007) (3) (b) and (4) (b), we have that

$$
\mathbb{E}\left[s^{Y(t)} \mid I(t)=1\right]=\mathbb{E}\left[s^{B(t)}\right]=\left\{\begin{array}{ll}
\frac{(\lambda-\gamma) \exp (\{\gamma-\lambda\} t)}{\lambda(1-s)-(\gamma-\lambda s) \exp (\{\gamma-\lambda\} t)} & (\lambda \neq \gamma) \\
\frac{1}{1+\lambda t(1-s)} & (\lambda=\gamma)
\end{array} .\right.
$$

Then since $\mathbb{P}(I(t)=1)=\exp (-\gamma t),(3.2)$ follows immediately from (3.3). 
For $s \in \mathbb{R}$ and $t \geq 0$, it is well-known (see, for example, Grimmett and Strizaker (1992), page 252) that

$$
\mathbb{E}\left[s^{Z(t)}\right]=\left\{\begin{array}{ll}
\frac{\gamma(1-s)-(\gamma-\lambda s) \exp (\{\gamma-\lambda\} t)}{\lambda(1-s)-(\gamma-\lambda s) \exp (\{\gamma-\lambda\} t)} & (\lambda \neq \gamma) \\
\frac{\lambda t+s\{1-\lambda t\}}{1+\lambda t(1-s)} & (\lambda=\gamma)
\end{array} .\right.
$$

We can also write

$$
\begin{aligned}
\mathbb{E}\left[s^{Z(t)}\right] & =\mathbb{E}\left[s^{Z(t)} 1_{\{I(t)=0\}}\right]+\mathbb{E}\left[s^{Z(t)} 1_{\{I(t)=1\}}\right] \\
& =\varphi_{0}(s ; t)+s \varphi_{1}(s ; t) .
\end{aligned}
$$

Then (3.1) follows straightforwardly by rearranging (3.5) and substituting in (3.4) and (3.2).

We now return to the household $(i, j)$. For $k=1,2, \ldots, i$, let $\left(I_{k}(t), Y_{k}(t)\right)$ be independent and identically distributed copies of $(I(t), Y(t))$. Then $\tilde{I}(\tau)=\sum_{k=1}^{i} I_{k}(\tau)$ and $\tilde{Y}(\tau)=\sum_{k=1}^{i} Y_{k}(\tau)$ denote the total number of infectives remaining in the household at the end of the morning and the total number of infectives at time $\tau$ who have been infected during the morning and can trace their infection back to a member of household $(i, j)$, respectively. With probability tending to 1 as $N \rightarrow \infty$, the $\tilde{Y}(\tau)$ individuals belong to distinct households and the probability that an infective belongs to a household of size $k$ is $\lim _{N \rightarrow \infty} q_{k}^{N}=q_{k}$. Therefore letting $\mathbf{X}_{(i, j)}^{M}$ denote the offspring distribution vector of a household starting a day with $i$ infectives and $j$ susceptibles at the end of the morning and $\phi_{(i, j)}^{M}(\mathbf{s}, \tau)$ denote the corresponding probability generating function, we have the following lemma.

Lemma 3.2. For $0 \leq s \leq 1$ and $\tau>0$,

$$
\phi_{(i, j)}^{M}(\mathbf{s}, \tau)=\sum_{m=0}^{i} s_{(m, j)}\left(\begin{array}{c}
i \\
m
\end{array}\right) \varphi_{0}\left(\sum_{h=1}^{\infty} q_{h} s_{(1, h-1)}, \tau\right)^{i-m} \varphi_{1}\left(\sum_{h=1}^{\infty} q_{h} s_{(1, h-1)}, \tau\right)^{m}
$$


Proof. First note that

$$
\begin{aligned}
\phi_{(i, j)}^{M}(\mathbf{s}, \tau) & =\mathbb{E}\left[\prod_{(k, l)} s_{(k, l)}^{X_{(i, j),(k, l)}^{M}}\right] \\
& =\sum_{m=0}^{i} \mathbb{E}\left[\prod_{(k, l)} s_{(k, l)}^{X_{(i, j),(k, l)}^{M}} 1_{\{\tilde{I}(\tau)=m\}}\right] \\
& =\sum_{m=0}^{i} s_{(m, j)} \mathbb{E}\left[\left(\sum_{h=1}^{\infty} q_{h} s_{(1, h-1)}\right)^{\tilde{Y}(\tau)} 1_{\{\tilde{I}(\tau)=m\}}\right],
\end{aligned}
$$

where $s_{(0, j)}=1$.

Exploiting the independence and exchangeability of the infectious behaviour of the $i$ initial infectives in the household during the morning period, we have that

$$
\begin{aligned}
& \mathbb{E}\left[\left(\sum_{h=1}^{\infty} q_{h} s_{(1, h-1)}\right)^{\tilde{Y}(\tau)} 1_{\{\tilde{I}(\tau)=m\}}\right] \\
= & \left(\begin{array}{c}
i \\
m
\end{array}\right) \mathbb{E}\left[\left(\sum_{h=1}^{\infty} q_{h} s_{(1, h-1)}\right)^{\sum_{k=1}^{i} Y_{k}(\tau)} \prod_{k=1}^{m} 1_{\left\{I_{k}(\tau)=1\right\}} \prod_{k=m+1}^{i} 1_{\left\{I_{k}(\tau)=0\right\}}\right] \\
= & \left(\begin{array}{c}
i \\
m
\end{array}\right) \mathbb{E}\left[\left(\sum_{h=1}^{\infty} q_{h} s_{(1, h-1)}\right)^{Y_{1}(\tau)} 1_{\left\{I_{1}(\tau)=1\right\}}\right]^{m}\left[\left(\sum_{h=1}^{\infty} q_{h} s_{(1, h-1)}\right)^{Y_{i}(\tau)} 1_{\left\{I_{i}(\tau)=0\right\}}\right]^{i-m} \\
= & \left(\begin{array}{c}
i \\
m
\end{array}\right) \varphi_{0}\left(\sum_{h=1}^{\infty} q_{h} s_{(1, h-1)}, \tau\right)^{i-m} \varphi_{1}\left(\sum_{h=1}^{\infty} q_{h} s_{(1, h-1)}, \tau\right)^{m} .
\end{aligned}
$$

The lemma follows by substituting (3.8) into (3.7).

We turn our attention to the nighttime. During the night the epidemics within the different households evolve independently and we consider the within household dynamics. Given a maximum household size of $H$, there are $H(H+1) / 2$ possible states for an infectious household. We define an infinitesimal transition matrix $G$ with $1+H(H+1) / 2$ rows and columns describing the transitions of the infectious status of a household amongst the $H(H+1) / 2$ possible infectious statuses of a household and a recovered state, which we denote state $\emptyset$, where the household no longer contains any infectives (the number of remaining susceptibles in the household is not important). We label the rows (columns) of $G$ by the type of infective households, as above, with row (column) $(i, j)$ coming before row (column) $(k, l)$ if $i<k$ or if $i=k, j<l$ with 
the final row (column) corresponding to the recovered state. Therefore we have that all elements of $G$ are 0 except for $i \in \mathbb{N}$ and $j \in \mathbb{Z}^{+}$,

$$
\begin{aligned}
G_{(i, j),(i+1, j-1)} & =i j \beta & & \text { (infection) } \\
G_{(i, j),(i-1, j)} & =i \gamma & & \text { (recovery } i>1) \\
G_{(1, j), \emptyset} & =\gamma & & \text { (recovery } i=1) \\
G_{(i, j),(i, j)} & =-i\{j \beta+\gamma\} & & \text { (leaving state }(i, j)) .
\end{aligned}
$$

Let $U=\exp ((1-\tau) G)$, the transition matrix for the infectious status of households during the course of one night. Then $U_{(i, j),(k, l)}$ is the probability that a household which at the start of the night is in state $(i, j)$ is in state $(k, l)$ at the end of the night, $1-\tau$ time units later.

Let

$$
f(\mathbf{s})=\sum_{(k, l)}\left\{\sum_{h=1}^{\infty} q_{h} U_{(1, h-1),(k, l)}\right\} s_{(k, l)},
$$

where $s_{\emptyset}=1$. (Note that the probability that the epidemic dies out starting from a disease-free household is 1.) Then we can combine the within-household evolution of the epidemic during the night with the birth-death dynamics of the morning, by substituting $f(\mathbf{s})$ given in (3.10) into (3.6), to give for $\lambda \neq \gamma$,

$$
\begin{aligned}
\phi_{(i, j)}(\mathbf{s}, \tau)= & \sum_{m=0}^{i}\left(\begin{array}{c}
i \\
m
\end{array}\right)\left\{\sum_{(k, l)} U_{(m, j),(k, l)} s_{(k, l)}\right\}\{(\lambda-\gamma) \exp (-\lambda \tau)\}^{m} \\
& \times \frac{\{\gamma(1-f(\mathbf{s}))-(\gamma-\lambda f(\mathbf{s})) \exp (\{\gamma-\lambda\} \tau)+f(\mathbf{s})(\gamma-\lambda) \exp (-\lambda \tau)\}^{i-m}}{\{\lambda(1-f(\mathbf{s}))-(\gamma-\lambda f(\mathbf{s})) \exp (\{\gamma-\lambda\} \tau)\}^{i}} .
\end{aligned}
$$

For $\lambda=\gamma$, we have that

$$
\begin{aligned}
\phi_{(i, j)}(\mathbf{s}, \tau)= & \sum_{m=0}^{i}\left(\begin{array}{c}
i \\
m
\end{array}\right)\left\{\sum_{(k, l)} U_{(m, j),(k, l)} s_{(k, l)}\right\}\{\exp (-\lambda \tau)\}^{m} \\
& \times \frac{\{\lambda t+f(\mathbf{s})\{1-\lambda t-\exp (-\lambda t)\}\}^{i-m}}{\{1+\lambda t(1-f(\mathbf{s}))\}^{m}} .
\end{aligned}
$$

We collect together the above results in the theorem below. 
Theorem 3.1. Let $\mathbf{s}=\left(s_{(1,0)}, s_{(1,1)}, \ldots, s_{(H, 0)}\right)$ denote the smallest solution in $[0,1]^{H(H+1) / 2}$ of

$$
\mathbf{s}=\phi(\mathbf{s}, \tau)
$$

where the $(i, j)^{t h}$ component of $\phi(\mathbf{s}, \tau)$ is $\phi_{(i, j)}(\mathbf{s}, \tau)$ given by $(3.11)(\lambda \neq \gamma)$ or $(3.12)$ $(\lambda=\gamma)$.

Then $1-s_{(i, j)}$ is the probability a major epidemic outbreak starting with an infectious household of type $(i, j)$ at the start of a day.

We briefly comment on Theorem 3.1 before studying some special cases in Section 4 . Firstly, we have not derived an explicit expression for $U^{*}$. However, it is straightforward to compute $U$ numerically and for $H$ small, it is possible to obtain $U$ by brute force, since there are at most $i+2 j$ transitions involving a household starting in state $(i, j)$. For example, for $H=2$, the $3 \times 3$ matrix $\tilde{U}$ defining transitions during the night between the states $\{(1,0),(1,1),(2,0)\}$ with $\beta \neq \gamma$ satisfies

$$
\tilde{U}=\left(\begin{array}{ccc}
\exp (-(1-\tau) \gamma) & 0 & 0 \\
A_{u} & \exp (-(\gamma+\beta)(1-\tau)) & B_{u} \\
2(\exp (-\gamma t)-\exp (-2 \gamma t)) & 0 & \exp (-2 \gamma t)
\end{array}\right)
$$

where

$$
\begin{aligned}
A_{u} & =\frac{2}{\gamma-\beta}\{(\gamma-\beta) \exp (-(1-\tau) \gamma)+\beta \exp (-2 \gamma(1-\tau))-\gamma \exp (-(1-\tau)(\gamma+\beta))\} \\
B_{u} & =\frac{\beta}{\beta-\gamma}(\exp (-2 \gamma(1-\tau))-\exp (-(\gamma+\beta)(1-\tau))) .
\end{aligned}
$$

Then it is trivial to obtain $U$ from $\tilde{U}$ since $\tilde{U}$ is the sub-matrix forming the first 3 rows and columns of $U$ with each row of $U$ summing to 1 . Secondly, for all $i \geq 1$, $s_{i, 0}=s_{1,0}^{i}$. This is because individuals belonging to households with no susceptibles behave independently. Therefore we can reduce the number of types in the branching process by $H-1$. However, it is often easier to work with the inclusion of types $(2,0), \ldots,(H, 0)$, for example, in the presentation of the probability generating function in (3.11) and (3.12). Thirdly, it is straightforward to extend the above to compute the extinction probability of the epidemic started at some point during the day with any configuration of initial infectives. We simply compute the probability generating 
function for the total number of infectious households of each type at the end of the first day. Fourthly, it is straightforward to allow for different recovery rates during the morning and night. This would be a departure from the assumption of independent and identically distributed infectious periods but may in some cases be more biologically reasonable. Similarly, by extending the number of types of individuals we could easily allow for different infection rates in different sized households. Finally, as mentioned earlier extensions to more general infectious period distributions using the method of stages (Barbour (1976), Lloyd (2001)) or an SEIR epidemic model with inclusion of a latent period are possible. The main complications are that the number of types grows exponentially with the number of stages and no generic expression for $\phi_{(i, j)}^{M}(\mathbf{s}, \tau)$ exists. However numerical computation of $\mathbf{s}$ is possible as demonstrated in Section 5.

\section{Special Cases}

\subsection{Homogeneously mixing epidemic with time of day effects}

Consider the case where $H=1$, all individuals live alone, or equivalently $\beta=0$, there is no within-household infection. In this case, we have a homogeneously mixing epidemic where the population alternates between being active and mixing (morning) and being passive and not mixing (night). During the night no infections can now take place but individuals can recover. Furthermore, we only require a single type of individual $(1,0)$ and correspondingly

$$
U=\left(\begin{array}{cc}
\exp (-(1-\tau) \gamma) & 1-\exp (-(1-\tau) \gamma) \\
0 & 1
\end{array}\right)
$$

Moreover, we do not need to distinguish between the individual (household) infected at the start of the day and those individuals infected during the morning as a result of the epidemic emanating from the initial infective. Hence $\mathbf{s}=s, \phi_{(1,0)}^{M}(s, \tau)=\mathbb{E}\left[s^{Z(\tau)}\right]$ and using (3.4) and (4.1), we have that

$$
\begin{aligned}
\phi_{(1,0)}(s, \tau) & =\mathbb{E}\left[z(s)^{Z(\tau)}\right] \\
& =\frac{\gamma(1-\exp (\{\gamma-\lambda\} \tau))+z(s)(\lambda \exp (\{\gamma-\lambda\} \tau)-\gamma)}{(\lambda-\gamma \exp (\{\gamma-\lambda\} \tau))+z(s)(\lambda \exp (\{\gamma-\lambda\} \tau)-\lambda)}
\end{aligned}
$$

where

$$
z(s)=1-\exp (-\gamma(1-\tau))+s \exp (-\gamma(1-\tau)) .
$$


Since $\phi_{(1,0)}(s, \tau)$ can be expressed in the form $(A+B s) /(C+D s)$, where $A+B=$ $C+D$, it is straightforward to show that the equation $\phi_{(1,0)}(s, \tau)=s$ can be expressed as a quadratic equation in $s$ with $s=1$ a solution. Hence, the extinction probability of the approximating branching process is

$$
s=\min \left\{1,-\frac{(\lambda-\gamma) \exp ((\gamma-\lambda) \tau)-\exp (-\gamma(1-\tau))(\lambda \exp ((\gamma-\lambda) \tau)-\gamma)}{\lambda \exp (-\gamma(1-\tau))(\exp ((\gamma-\lambda) \tau)-1)}\right\} .
$$

The basic reproduction number, $R_{0}$, of the branching process is given by

$$
R_{0}=\left.\frac{d}{d s} \phi_{(1,0)}(s, \tau)\right|_{\{s=1\}} .
$$

It is trivial to show that

$$
\begin{aligned}
R_{0} & =\frac{\exp (-\gamma(1-\tau))(\lambda \exp ((\gamma-\lambda) \tau)-\gamma)-\lambda \exp (-\gamma(1-\tau))(\exp ((\gamma-\lambda) \tau)-1)}{\{(\lambda-\gamma) \exp ((\gamma-\lambda) \tau)-\lambda \exp (-\gamma(1-\tau))(\exp ((\gamma-\lambda) \tau)-1)\}+\lambda \exp (-\gamma(1-\tau))(\exp ((\gamma-\lambda) \tau)-1)} \\
& =\frac{(\lambda-\gamma) \exp (-\gamma(1-\tau))}{(\lambda-\gamma) \exp ((\gamma-\lambda) \tau)}=\exp (\lambda \tau-\gamma) .
\end{aligned}
$$

Therefore the epidemic is supercritical if $R_{0}>1$, which occurs if and only if $\lambda \tau>\gamma$. (The infection rate in the morning times the length of the morning period is greater than the recovery rate.)

For $0 \leq t<1$, let $s_{t}$ denote the extinction probability if the initial infective starts their infectious period at time $t$ with $s_{0}=s$. It is straightforward to show that

$$
\begin{aligned}
s_{t} & =\mathbb{E}\left[z(s)^{Z(\tau-t)}\right] \quad(0 \leq t<\tau) \\
& =\frac{\gamma(s-1) \exp (-(1-\gamma) \tau)-(\gamma-\lambda z(s)) \exp (\{\gamma-\lambda\}(\tau-t))}{\lambda(s-1) \exp (-(1-\gamma) \tau)-(\gamma-\lambda z(s)) \exp (\{\gamma-\lambda\}(\tau-t))} \\
s_{t} & =1-\exp (-\gamma(1-t))+\exp (-\gamma(1-t)) s \quad(\tau \leq t<1) .
\end{aligned}
$$

Furthermore, if the epidemic is supercritical $\left(R_{0}>1, \lambda>\gamma / \tau, s<1\right)$, we have that $s_{t}$ is increasing on $[0, \tau)$ and decreasing on $[\tau, 1)$.

Finally, it is interesting to investigate the effect of varying the length of the morning, $\tau$, whilst keeping $\lambda \tau\left(R_{0}\right)$ fixed. For $0<\tau \leq 1$, let $\check{s}_{\tau}^{M}$ denote the extinction probability of the epidemic process with a morning period of length $\tau$ and $\lambda=\check{\lambda} / \tau$. Then provided $\check{\lambda}>\gamma$, the epidemic is supercritical and $R_{0}=\exp (\check{\lambda}-\gamma)$ is constant, independent of $\tau$. From (4.4), we have that

$$
\begin{aligned}
\check{s}_{\tau}^{M} & =\frac{\check{\lambda}\{\exp (\gamma-\check{\lambda})-\exp (\gamma \tau-\check{\lambda})\}+\gamma \tau\{1-\exp (\gamma-\check{\lambda})\}}{\check{\lambda}\{1-\exp (\gamma \tau-\check{\lambda})\}} \\
& =1+\frac{1-\exp (\gamma-\check{\lambda})}{\check{\lambda}} \times \frac{\gamma \tau-\check{\lambda}}{1-\exp (\gamma \tau-\check{\lambda})} .
\end{aligned}
$$


Since $x /(1-\exp (x))$ is an increasing function on $(-\infty, 0)$, it follows from (4.9) that $\check{s}_{\tau}^{M}$ increases as $\tau$ increases on the range $[0,1]$. Now suppose that the initial infective enters the population at the start of the night, so spends the first $1-\tau$ units of its infectious period in isolation. Let $\check{s}_{\tau}^{N}$ denote the corresponding extinction probability of the approximating branching process. Then

$$
\begin{aligned}
\check{s}_{\tau}^{N}=z\left(\check{s}_{\tau}^{M}\right) & =1-\exp (-\gamma(1-\tau))+\exp (-\gamma(1-\tau)) \check{s}_{\tau}^{M} \\
& =1+\exp (-\gamma(1-\tau)) \frac{1-\exp (\gamma-\check{\lambda})}{\check{\lambda}} \times \frac{\gamma \tau-\check{\lambda}}{1-\exp (\gamma \tau-\check{\lambda})} \\
& =1+\frac{\exp (-\gamma)-\exp (-\check{\lambda})}{\lambda \exp (-\check{\lambda})} \times \frac{(\gamma \tau-\check{\lambda}) \exp (\gamma \tau-\check{\lambda})}{1-\exp (\gamma \tau-\check{\lambda})} .
\end{aligned}
$$

Since $x \exp (x) /(1-\exp (x))$ is a decreasing function on $(-\infty, 0)$, it follows from (4.10) that $\check{s}_{\tau}^{N}$ decreases as $\tau$ increases on the range $[0,1]$, the converse of the result for $\check{s}_{\tau}^{M}$.

\section{2. $\beta=\infty$}

The opposite extreme case is when the epidemic is highly contagious within households. In particular, $\beta=\infty$ corresponds to an infective on returning to their household immediately infecting any susceptibles within the household. In this case the total number of infectives at the end of a night where at the start of the night an infective infects the other $h-1$ individuals in a household of size $h$ follows a binomial distribution with parameters $h$ and $\exp (-\gamma(1-\tau))$. Therefore since there will be no susceptibles in any infectious household at the end of each day, the possible infectious statuses of households are $(i, 0)(i=1,2, \ldots)$. Using the second observation after Theorem 3.1 , that a household with $i$ infectives and 0 susceptibles has the same behaviour as $i$ independent households with 1 infectives and 0 susceptibles, we can, as noted in Section 4.1, reduce the branching process approximation to a single type branching process.

We have the following corollary to Theorem 3.1.

Corollary 4.1. For $\beta=\infty, s_{(1,0)}$ is the smallest solution in $[0,1]$ of

$$
s_{(1,0)}=\varphi\left(s_{(1,0)}, \tau\right),
$$

where $z\left(s_{(1,0)}\right)$ is defined in (4.3) and

$$
\varphi\left(s_{(1,0)}, \tau\right)=\varphi_{0}\left(\sum_{h=1}^{\infty} q_{h} z\left(s_{(1,0)}\right)^{h}, \tau\right)+z\left(s_{(1,0)}\right) \varphi_{1}\left(\sum_{h=1}^{\infty} q_{h} z\left(s_{(1,0)}\right)^{h}, \tau\right) .
$$


Proof. We construct the single-type branching process as follows. Consider a single infective who belongs to a household with no susceptible individuals, type $(1,0)$, at the start of the morning. From Lemma 3.2, (3.6), we have that

$$
\phi_{(1,0)}^{M}(\mathbf{s}, \tau)=\left\{\varphi_{0}\left(\sum_{h=1}^{\infty} q_{h} s_{(1, h-1)}, \tau\right)+s_{1,0} \varphi_{1}\left(\sum_{h=1}^{\infty} q_{h} s_{(1, h-1)}, \tau\right)\right\} .
$$

Now since $s_{(k, 0)}=s_{(1,0)}^{k}(k=1,2, \ldots), U_{(1, h-1),(k, 0)}^{*}=\left(\begin{array}{l}h \\ k\end{array}\right) \exp (-\gamma(1-\tau))^{k}(1-$ $\exp (-\gamma(1-\tau)))^{h-k}(k=0,1, \ldots, h)$ and $U_{(1, h-1),(k, l)}^{*}=0$ otherwise, we have that $f(\mathbf{s})$ in (3.10) becomes

$$
\begin{aligned}
f(\mathbf{s}) & =\sum_{(k, l)}\left\{\sum_{h=1}^{\infty} q_{h} U_{(1, h-1),(k, l)}^{*}\right\} s_{(k, l)} \\
& =\sum_{h=1}^{\infty} q_{h} \sum_{k=0}^{h} U_{(1, h-1),(k, 0)}^{*} s_{(k, 0)} \\
& =\sum_{h=1}^{\infty} q_{h} \sum_{k=0}^{h}\left(\begin{array}{l}
h \\
k
\end{array}\right) \exp (-\gamma(1-\tau))^{k}(1-\exp (-\gamma(1-\tau)))^{h-k} s_{1,0}^{k} \\
& =\sum_{h=1}^{\infty} q_{h} z\left(s_{(1,0)}\right)^{h} .
\end{aligned}
$$

We then substitute $f(\mathbf{s})$ and $z\left(s_{(1,0)}\right)$ in for $\sum_{h=1}^{\infty} q_{h} s_{(1, h-1)}$ and $s_{1,0}$, respectively, in (4.13) to give (4.12) as required.

The probability of extinction of the epidemic given by (4.11) will depend upon the distribution of household sizes, $\lambda, \gamma$ and $\tau$. By contrast the basic reproduction number $R_{0}$ which satisfies

$$
R_{0}=\left.\frac{d}{d s} \varphi(s, \tau)\right|_{s=1}=\frac{1+\{\exp (\lambda \tau)-1\} \sum_{h=1}^{\infty} h q_{h}}{\exp (\gamma)},
$$

only depends upon the mean size of a household, $\lambda \tau$ and $\gamma$. For comparison the household epidemic model of Ball et al. (1997) with global infection rate $\lambda_{G}=\lambda \tau$ and local infection rate $\lambda_{L}=\beta(1-\tau)=\infty$ has a basic reproduction number $R_{*}=$ $\lambda \tau \sum_{h=1}^{\infty} h q_{h} / \gamma$. Note that there are multiple candidates for the basic reproduction number of household epidemic, see Pellis et al. (2012), all coinciding at $R_{*}=1$. We take $R_{*}$ to be the basic reproduction number defined in Ball et al. (1997) corresponding to the global infection rate times the mean size of a household epidemic. The parameters $\lambda_{G}$ and $\lambda_{L}$ are chosen to ensure that both epidemic models have the same mean number 
of community and household contacts per individual per day. Whilst $R_{*}$ and $R_{0}$ are not directly comparable, we note that for $R_{*}=1$ (the household epidemic model of Ball et al. (1997) is critical), $R_{0}<1$ unless $q_{1}=1$, in which case $R_{0}=1$.

\section{Numerics and extensions}

In general analytical expressions are not available for $\mathbf{s}$. However we can solve (3.13) numerically for the SIR model with exponential infectious periods. This is easily done by initialising with $\mathbf{s}^{0}=(0, \ldots, 0)$ with $s_{\emptyset}=1$ and iteratively setting $\mathbf{s}^{k}=\phi\left(\mathbf{s}^{k-1}, \tau\right)$, stopping when $\mathbf{s}^{k}$ and $\mathbf{s}^{k-1}$ agree to some predefined precision. (We stopped when the difference in the $L_{1}$-norm fell below $10^{-5}$.)

In Table 1 we present the probability of extinction for 45 parameter combinations in a population consisting of households of size 4 . The parameter combinations are achieved by combining 5 different choices of $\gamma,(6,2,1,1 / 3,1 / 7)$, with three different choices of $(\lambda, \beta)$, namely $\gamma(1.1,5), \gamma(2,2)$ and $\gamma(3,1)$, and three different choices of $\tau=0.25,0.5,0.75$. Thus we consider mean infectious periods ranging from 4 hours to 1 week and that the ratio between the infection and recovery parameters are kept constant as $\gamma$ varies. Also the lengths of the morning periods are 6, 12 or 18 hours. For comparison we report the extinction probability of a standard household epidemic with rates $(\tau \lambda,(1-\tau) \beta, \gamma)$. Thus in both models an individual makes the same number of global and local infectious contacts per day. Note that for the standard household model the extinction probability for parameters $c(\tau \lambda,(1-\tau) \beta, \gamma)$ is the same for all $c>0$. From Table 1 we observe that the extinction probability is generally higher for short infectious periods and unsurprisingly as $\gamma$ tends to 0 the extinction probabilities converge to those obtained for the standard household model. The largest differences in the extinction probabilities are observed when $\beta$ is large and/or $\tau$ is small, which are the cases when the household dynamics are of greatest importance. Similar results are observed with different household sizes and parameter combinations. 


\begin{tabular}{|c|c|c|c|c|c|c|c|c|c|}
\hline & \multicolumn{3}{|c|}{$\tau=0.25$} & \multicolumn{3}{c|}{$\tau=0.5$} & \multicolumn{3}{c|}{$\tau=0.75$} \\
\cline { 2 - 9 }$\gamma$ & $\gamma(1.1,5)$ & $\gamma(2,2)$ & $\gamma(3,1)$ & $\gamma(1.1,5)$ & $\gamma(2,2)$ & $\gamma(3,1)$ & $\gamma(1.1,5)$ & $\gamma(2,2)$ & $\gamma(3,1)$ \\
\hline 6 & 1.000 & 1.000 & 0.957 & 1.000 & 0.598 & 0.340 & 0.927 & 0.502 & 0.333 \\
2 & 1.000 & 0.872 & 0.553 & 0.848 & 0.497 & 0.357 & 0.688 & 0.470 & 0.335 \\
1 & 1.000 & 0.642 & 0.503 & 0.623 & 0.423 & 0.359 & 0.543 & 0.429 & 0.337 \\
$\frac{1}{3}$ & 1.000 & 0.553 & 0.495 & 0.451 & 0.366 & 0.362 & 0.413 & 0.387 & 0.338 \\
$\frac{1}{7}$ & 0.977 & 0.544 & 0.498 & 0.415 & 0.352 & 0.363 & 0.378 & 0.375 & 0.338 \\
$\mathrm{~S}$ & 0.968 & 0.542 & 0.502 & 0.398 & 0.344 & 0.365 & 0.355 & 0.365 & 0.339 \\
\hline
\end{tabular}

Table 1. Extinction probabilities for combinations of $(\lambda, \beta, \gamma, \tau)$. The final row with $\gamma=S$ corresponds to the standard household model.

The time of day at which the initial infective can have a dramatic effect on the extinction probability. For example, for the case $\gamma=6$ with $(\lambda, \beta)=\gamma(3,1)$ and $\tau=0.5$ the extinction probability is 0.862 if the initial infective enters the population at the start of the night compared with 0.339 if the initial infective enters the population just before the end of the night. The difference is less dramatic as $\gamma$ decreases. During the morning period the extinction probability is either monotonically increasing or decreasing depending upon whether or not time in the household helps or hinders the epidemics progress. During the night the extinction probability is either monotonic exhibiting the opposite behaviour to the morning or reaches a minimum at some time point during the middle of the night. For example, for $(\lambda, \beta, \gamma)=(2,2,1)$ and $\tau=0.5$, the extinction probabilities for the initial infective entering the population at times $t=0.5,0.7,1.0$ are $0.358,0.340,0.423$, respectively.

Although it is not possible in general to obtain an explicit expression for $\phi(\mathbf{s}, \tau)$, the approach taken in Section 3 can be extended to obtain numerical expressions for $\phi(\mathbf{s}, \tau)$ for the SEIR model and more general SIR models. Explicitly we can use the method of stages, Barbour (1976), and allow individuals once infected to go through a number of stages before recovering from the disease. That is, there are $k$ stages with an individual's time spent in stage $l$ being exponentially distributed with mean $1 / \gamma_{l}$ and the individual having infectious rates $\lambda_{l}$ and $\beta_{l}$ in the community and household, respectively. By keeping track of the number of individuals in each stage it is possible, 
as in Section 3, to construct an infinitesimal matrix $G$ for the dynamics of the epidemic in a household during the night and to obtain $U=\exp ((1-\tau) G)$, the transition matrix for the infectious status of a household during the course of one night. Let $\mathbf{i}=\left(i_{1}, \ldots, i_{k}, j\right) \in\{\mathbf{N} \cup\{0\}\}^{k+1}$ and define a household to be in state $\mathbf{i}$ if there are $i_{r}$ individuals in state $r(1 \leq r \leq k)$ and $j$ susceptibles. Suppose that we start with a single household in state $\mathbf{i}$. After a small amount of time, $\Delta t$ say, at most one event will have occurred with probability $1-o(\Delta t)$ with either an individual within the household transiting to the next stage or an infection taking place. For $r=1,2, \ldots, k-1$, let $\mathbf{w}_{r}$ be a vector of length $k+1$ with the $r^{t h}$ and $r+1^{\text {st }}$ elements being -1 and 1 , respectively, let $\mathbf{w}_{k}$ be a vector of length $k+1$ with $k^{\text {th }}$ element equal to -1 and let $\mathbf{v}$ be a vector length $k+1$ with the first and last elements equal to 1 and $h-1$, respectively, with all other elements in the vectors equal to 0 . Therefore it is straightforward to show that $\phi_{\mathbf{i}}^{M}(\mathbf{s}, t)$ satisfies the Kolmogorov backward equations,

$$
\frac{\partial \phi_{\mathbf{i}}^{M}(\mathbf{s}, t)}{\partial t}=\sum_{r=1}^{k} \gamma_{k}\left\{\phi_{\mathbf{i}+\mathbf{w}_{r}}^{M}(\mathbf{s}, t)-\phi_{\mathbf{i}}^{M}(\mathbf{s}, t)\right\}+\left(\sum_{r=1}^{k} \lambda_{r} i_{r}\right) \phi_{\mathbf{i}}^{M}(\mathbf{s}, t)\left\{\phi_{\mathbf{v}}^{M}(\mathbf{s}, t)-1\right\},
$$

where $\phi_{1}^{M}(\mathbf{s}, t)$ is the offspring distribution probability generating function for a period of length $t$ during the morning initiated by a single infectious household in state $\mathbf{l}$ at time 0. Note that $\phi_{\mathbf{i}+\mathbf{w}_{k}}^{M}(\mathbf{s}, t)=1$ if the epidemic dies out in the household. Then (5.1) can be solved numerically using Euler's method, to obtain $\phi^{M}(\mathbf{s}, \tau)$ and combined with $U, \phi(\mathbf{s}, \tau)=\phi^{M}(U \mathbf{s}, \tau)$.

Whilst the number of types grow rapidly with $k$ and the household size $h$, it is practical to compute $\phi(\mathbf{s}, \tau)$ for small $k$. For $k=2$, we obtain the SEIR model if we set $\lambda_{1}=\beta_{1}=0$ and the SIR model with a $\operatorname{Gamma}\left(2, \gamma_{1}\right)$ infectious period if we set $\lambda_{1}=\lambda_{2}, \beta_{1}=\beta_{2}$ and $\gamma_{1}=\gamma_{2}$. Comparing the probability of extinction for the SIR models with infectious periods $\operatorname{Gamma}(2,2 \gamma)$ and $\operatorname{Exp}(\gamma)$ and common infection rates $\lambda$ and $\beta$ produce interesting results. For standard household epidemic models $\operatorname{Exp}(\gamma)$ has the larger extinction probability and this is usually the case when time of day effects are included. However, there are exceptions when the within household infection rate is low (prohibitive to the epidemics progress) in which case the increased variability of the exponential distribution assists the epidemics survival. An example 
is $\lambda=2.5, \beta=0$ and $\gamma=1$, where the extinction probabilities are 0.808 and 0.800 for $\operatorname{Gamma}(2,2 \gamma)$ and $\operatorname{Exp}(\gamma)$ infectious periods, respectively. Similarly the presence of a latent period can either assist or hinder the survival of the epidemic. The extinction probability does not always vary in a monotonic manner with the length of the latent period. For example, for the SEIR model with infection rates $\lambda=2.5, \beta=0$ and $\operatorname{Exp}(1)$ infectious periods, the extinction probabilities are $0.758,0.776,0.814$ and 0.800 for $\gamma_{1}=0.1,1,5$ and infinity, respectively, with the latter case corresponding to the SIR model. The reason for this behaviour is that more individuals are infected at the end of the morning than the beginning as the epidemic grows and thus a short, but non-zero, latent period means that many infectives start their infectious period early in the night when they can't infect anybody. The inclusion of a latent period, if it is not very short, averages out the varability in the extinction probability for different introductory times of the initial infective. Thus in conclusion, the impact of time of day effects on the extinction probability are often difficult to predict and can be non-negligible, so require due consideration.

\section{Acknowledgements}

I would like to thank an anonymous referee for their thought provoking comments which motivated Section 5 .

\section{References}

Aldous, D. J. (1985) Exchangeability and related topics. In Ecole d'Été de Probabilités de Saint-Flour XIII, 1993 (Lecture Notes in Mathematics 1117), ed. Hennequin, P. L. Springer, Berlin.

Ball, F.G. (1983) The threshold behaviour of epidemic models. J. Appl. Prob. 20, $227-241$

Ball, F.G. (1996) Threshold behaviour in stochastic epidemics among households. In: Athens Conference on Applied Probability and Time Series, Volume I: Applied Probability (eds. C.C. Heyde, Y.V. Prohorov, R. Pyke and S.T. Rachev), Lecture Notes in Statistics 114, 253-266. 
Ball, F.G. and Donnelly, P. (1995) Strong approximations for epidemic models. Stoch. Proc. Appl., 55, 1-21.

Ball, F.G., Mollison, D. and Scalia-Tomba, G. (1997) Epidemics with two levels of mixing. Ann. Appl. Prob. 7, 46-89.

Ball, F.G. and Neal, P.J. (2002) A general model for stochastic SIR epidemics with two levels of mixing. Math. Biosci. 180, 73-102.

Ball, F.G. and Neal, P.J. (2003) The great circle epidemic model. Stoch. Proc. Appl. 107, 233-268.

Ball, F.G. and Neal, P.J. (2008) Network epidemic models with two levels of mixing. Math. Biosci. 212, 69-87.

Barbour, A.D. (1976) Network of queues and the method of stages. Adv. Appl. Prob., 8, 584-591.

Gani, J. and Stals, L. (2007) A note on three stochastic processes with immigration. ANZIAM J. 48, 409-418.

Grimmett, G.R. and Strizaker, D.R. (1992) Probability and Random Processes. Second edition. Clarendon Press, Oxford.

Lamperti, J. (1967) Continuous state branching processes. Bull. Amer. Math. Soc. 73, $382-386$.

Lloyd, A.L. (2001) Destabilization of epidemic models with the inclusion of realistic distributions of infectious periods. Proceedings of the Royal Society of London. Series B: Biological Sciences, 268, 985-993.

Pellis, L., Ball, F. and Trapman, P. (2012) Reproduction numbers for epidemic models with households and other social structures. I. Definition and calculation of $R_{0}$. Math. Biosci. 235, 85-97.

Whittle, P. (1955) The outcome of a stochastic epidemic - a note on Bailey's paper. Biometrika, 42, 116-122. 